\title{
Study of load-bearing timber-wall elements using experimental testing and mathematical modelling
}

\author{
Premrov, M. ${ }^{a}$, Ber, B. ${ }^{b}$, Kozem Šilih, E. ${ }^{a,{ }^{*}}$ \\ aniversity of Maribor, Faculty of Civil Engineering, Transportation Engineering and Architecture, Maribor, Slovenia \\ belovica hiše d.o.o., Preddvor, Slovenia
}

\section{A B S T R A C T}

Combining timber and glass in the wall elements of the building envelope with the proper orientation of such transparent façade elements enables the utilization of solar energy for heating and internal illumination of the building. However, the asymmetrical layout of timber-glass wall elements in such buildings can result in problems with the horizontal stability of the structure, so their participation to load-bearing capacity of the structure is usually neglected. The study deals with solutions for such elements as horizontal loadbearing members with proper connection details. First, specifically developed timber-glass wall elements were experimentally tested under monotonic and cyclic horizontal point load, and further in combination with classical timberframed wall elements implemented into special single and two-storey boxhouse models, which were further experimentally tested on the shaking table. In the second part as the main goal of the study, a quite simple mathematical model of the box-house prototypes is developed using a fictive diagonal element for simulating the racking stiffness of the bracing timber-glass wall element. The calculated results for the $1^{\text {st }}$ vibration period are compared with the previously measured experimental results to prove an accuracy of the developed model. Finally, a linear time-history calculation is done as a sample presentation of the developed mathematical model using Landers acceleration spectrum. The developed mathematical model enables a simple and effective seismic response calculation of timber buildings considering the developed timber-glass wall elements as load-bearing bracing elements against horizontal load actions. The model can also be recommended for using in further parametric numerical academic studies analysing the influence of various parameters.
\end{abstract}

\author{
ARTICLE INFO \\ Keywords: \\ Wall elements; \\ Timber; \\ Timber-glass building; \\ Stiffness; \\ Vibrations; \\ Experiments; \\ Modelling; \\ Landers accelerogram \\ *Corresponding author: \\ erika.kozem@um.si \\ (Kozem Šilih, E.) \\ Article history: \\ Received 6 October 2020 \\ Revised 26 February 2021 \\ Accepted 7 March 2021

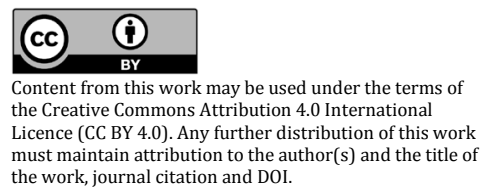 \\ must maintain attribution to the author(s) and the work \\ the work, journal citation and DOI.
}

\section{References}

[1] Diakaki, C., Grigoroudis, E., Kabelis, N., Kolokotsa, D., Kalaitzakis, K., Stavrakakis, G. (2010). A multi-objective decision model for the improvement of energy efficiency in buildings, Energy, Vol. 35, No.12, 5483-5496, doi: 10.1016/j.energy.2010.05.012.

[2] Olabi, A.G. (2013). State of the art on renewable and sustainable energy, Energy, Vol. 61, 2-5, doi: 10.1016/ j.energy.2013.10.013.

[3] Olabi, A.G. (2014). 100\% sustainable energy, Energy, Vol. 77, 1-5, doi: 10.1016/i.energy.2014.10.083.

[4] Lund, H., Mathiesen, BV. (2009). Energy system analysis of $100 \%$ renewable energy systems - The case of Denmark in years 2030 and 2050, Energy, Vol. 34, No. 5, 524-531, doi: 10.1016/j.energy.2008.04.003.

[5] Lund, H., Möller, B., Mathiesen, B.V., Dyrelund, A. (2010). The role of district heating in future renewable energy systems, Energy, Vol. 35, No. 3, 1381-1390, doi: 10.1016/i.energy.2009.11.023.

[6] Itard, L.C.M., Meijer, F. (2008). Towards a sustainable Northern European housing stock: Figures, facts and future, The series Sustainable Urban Areas, IOS Press, Vol. 22, Amsterdam, The Netherlands.

[7] Wurm, J. (2007). Glass structures - Design and construction of self-supporting skins, Birkhäuser Verlag AG, Basel, Boston, Berlin, doi: 10.1007/978-3-7643-8317-6. 
[8] Žegarac Leskovar, V., Premrov, M. (2013). Energy-efficient timber-glass houses, Springer, London, Heidelberg, New York, Dordrecht, doi: 10.1007/978-1-4471-5511-9.

[9] Premrov, M., Žegarac Leskovar, V., Mihalič, K. (2016). Influence of the building shape on the energy performance of timber-glass buildings in different climatic conditions, Energy, Vol. 108, 201-211, doi: 10.1016/i.energy.2015. 05.027.

[10] Žegarac Leskovar, V., Premrov, M. (2011). An approach in architectural design of energy-efficient timber buildings with a focus on the optimal glazing size in the south-oriented façade, Energy and Buildings, Vol. 43, No. 12, 3410-3418, doi: 10.1016/j.enbuild.2011.09.003.

[11] European Committe for Standardization CEN/TC 250 (2005). EN 1998-1:2005 Eurocode 8: Design of Structures for Earthquake Resistance - Part 1, General rules, seismic actions and rules for buildings, Brussels.

[12] European Committe for Standardization CEN/TC 250/SC5 N173 (2005). EN 1995-1-1:2005 Eurocode 5: Design of Timber Structures - Part 1-1, General rules and rules for buildings, Brussels.

[13] Antolinc, D., Rajčić, V., Žarnić, R. (2014). Analysis of hysteretic response of glass infilled wooden frames, Journal of Civil Engineering \& Management, Vol. 20, No. 4, 600-608, doi: 10.3846/13923730.2014.899265.

[14] Žarnić, R., Rajčić, V., Kržan, M. (2020). Response of laminated glass-CLT structural components to reverse lateral loading, Construction and Building Materials, Vol. 235, 1-17, doi: 10.1016/i.conbuildmat.2019.117509.

[15] Premrov, M., Serrano, E., Winter, W., Fadai, A., Nicklisch, F., Dujič, B., Šušteršič, I., Brank, B., Štrukelj, A., Držečnik, M., Buyuktaskin, H.A., Erol, G., Ber, B. (2012-2014). Workshop report "WP 6: Testing on life-size specimen components: shear walls, beams and columns including long-term behaviour". Woodwisdom-net, research project, load bearning timber-glass-composites, https://plus.si.cobiss.net/opac7/bib/18802454.

[16] Blyberg, L. (2011). Timber/glass adhesive bonds for structural applications, Licentiate thesis, Linnaeus University, School of Engineering, Sweden, http://www.diva-portal.org/smash/get/diva2:447937/FULLTEXT01.pdf.

[17] Blyberg, L., Serrano, E., Enquist, B., Sterley, M. (2012). Adhesive joints for structural timber/glass applications: Experimental testing and evaluation methods, International Journal of Adhesion and Adhesives, Vol. 35, 76-87, doi: 10.1016/j.ijadhadh.2012.02.008.

[18] Cruz, P.J.S., Pacheco, J.A.L., Pequeno, J.M.B. (2007). Experimental studies on structural timber glass adhesive bonding, In: COST E34, Bonding of Timber, 4th Workshop "Practical Solutions for Furniture \& Structural Bonding", Golden Bay Beach Hotel, Larnaca, Cyprus, https://sigarra.up.pt/feup/pt/conteudos service.conteudos cont?pct id=30543\&pv cod=41slaqqt89ra.

[19] Stepinac, M., Rajčić, V., Žarnić R. (2016). Timber-structural glass composite systems in earthquake environment, Gradjevinar, Vol. 68, No. 3, 211-219, doi: 10.14256/ICE.1505.2015.

[20] Dimona, S., Pinto, A., Feldmann, M., Denton, S. (2014). European Comission JCR Scientific and Policy Reports. Guidance for European Structural Design of Glass Components, Support to the implementation, harmonization and further development of the Eurocodes, Report EUR 26439 EN, Luxemburg, Publications Office of the European Union.

[21] Kozem Šilih, E., Premrov, M., Šilih, S. (2012). Numerical analysis of timber-framed wall elements coated with single fibre-plaster boards, Engineering Structures, Vol. 41, 118-125, doi: 10.1016/i.engstruct.2012.03.044.

[22] Šupek, S., Dujič, B., Žarnić, R. (2008). Influence of openings on shear capacity of KLH walls, In: Zbornik 30. zborovanja gradbenih konstrukterjev, Bled, Ljubljana: Slovensko društvo gradbenih konstruktorjev, Slovenia, $145-154$

[23] Kozłowski, M., Dorn, M., Serrano, E. (2015). Experimental testing of load-bearing timber-glass composite shear walls and beams. Wood Material Science \& Engineering, Vol. 10, No. 3 (Special issue), 276-286, doi: 10.1080/ 17480272.2015 .1061595$.

[24] Dorn, M., Kozłowski, M., Serrano, E., Enquist, B. (2014). Development of large-scale load-bearing timber-glass structural elements, In: Salenikovich, A. (ed.), World Conference on Timber Engineering, WCTE 2014, Quebec City, Canada.

[25] Hochhauser, W. (2011). Ein Beitrag zur Berechnung und Bemessung von geklebten und geklotzten Holz-GlasVerbundscheiben, Doctoral thesis, Vienna University of Technology, Austria, https://www.ub.tuwien.ac.at/diss/AC07810717.pdf.

[26] Štrukelj, A., Ber, B., Premrov, M. (2015). Racking resistance of timber-glass wall elements using different types of adhesives, Construction and Building Materials, Vol. 93, 130-143, doi: 10.1016/i.conbuildmat.2015.05.112.

[27] Ber, B., Finžgar, G., Premrov, M., Štrukelj A. (2018). On parameters affecting the racking stiffness of timber-glass walls, Glass Structures and Engineering, Vol. 4, No. 1, 69-82, doi: 10.1007/s40940-018-0086-5.

[28] Ber, B., Šušteršič, I., Premrov, M., Štrukelj, A., Dujič, B. (2015). Testing of timber-glass composite walls, Proceedings of the Institution of Civil Engineers - Structures and Buildings, Vol. 168, No.7, 500-513, doi: 10.1680/stbu.13. $\underline{00105}$.

[29] European Committe for Standardization CEN/TC 250/SC5 N173 (2011). EN 594:2011 Timber structures. Test methods. Racking strength and stiffness of timber frame wall panels, BSI, London, UK.

[30] Niedermaier, P. (2003). Shear-strength of glass panel elements in combination with timber frame constructions, In: Proceedings of the 8th International Conference on Architectural and Automotive Glass (GPD), Tampere, Finland, 262-264.

[31] European Committe for Standardization (2002). EN 12512:2002 Timber structures - Test methods - Cyclic testing of joints made with mechanical fasteners, https://standards.iteh.ai/catalog/standards/sist/82e23cd2-e11c-40ad-8ca0-384618804f92/sist-en-12512-2002.

[32] Pintarič, K., Premrov, M. (2013). Mathematical modelling of timber-framed walls using fictive diagonal elements, Applied Mathematical Modelling, Vol. 37, No. 16-17, 8051-8059, doi: 10.1016/j.apm.2013.02.050.

[33] Vogrinec, K., Premrov, M. (2018). Influence of the design approach on the behaviour of timber-frame panel buildings under horizontal forces, Engineering structures, Vol. 175, 1-12, doi: 10.1016/i.engstruct.2018.08.014. 
[34] Ber, B. (2015). Tragendes Glaswandelement für leichte Holzbauten: CH707540 (B1), 2015-01-15. Bern: EPO, SI P201300028, 2013-02-06; CH707540 (A2), 2014-08-15; SI23931 (A), 2013-05-31; DE102013217209 (A1), 201408-07; ITRM20130609 (A1), 2014-08-07. 


\title{
Študija nosilnih lesenih zidnih elementov z uporabo eksperimentalnega testiranja in matematičnega modeliranja
}

\author{
Premrov, M. ${ }^{a}$, Ber, B. ${ }^{b}$, Kozem Šilih, E. ${ }^{a,}{ }^{*}$ \\ aUniversity of Maribor, Faculty of Civil Engineering, Transportation Engineering and Architecture, Maribor, Slovenia \\ bJelovica hiše d.o.o., Preddvor, Slovenia
}

\section{POVZETEK}

Kombinacija lesa in stekla v stenskih elementih ovoja stavbe s pravilno orientacijo tovrstnih prosojnih fasadnih elementov omogoča izkoriščanje sončne energije za ogrevanje in notranjo osvetlitev objekta. Asimetrična postavitev leseno-steklenih stenskih elementov v takih objektih pa lahko povzroči probleme s horizontalno stabilnostjo konstrukcije, zato njihov vpliv na nosilnost konstrukcije običajno ni upoštevan. Študija obravnava rešitve za te elemente, kot so horizontalni nosilni elementi z ustreznimi povezavami. Najprej so bili posebej razviti leseno-stekleni stenski elementi eksperimentalno preizkušeni pod monotono in ciklično horizontalno točkovno obremenitvijo, nato pa $\mathrm{v}$ kombinaciji s klasičnimi leseno-okvirnimi stenskimi elementi implementirani v posebne eno- in dvonadstropne makete hiš, ki so bile nadalje eksperimentalno testirane na stresalni mizi. V drugem delu študije je bil kot glavni cilj raziskave razviti matematični model za opis prototipov maket hiš, v katerem za simulacijo togosti leseno-steklenega stenskega elementa uporabimo fiktivni diagonalni element. Izračunane rezultate za prvo nihajno periodo smo primerjali s predhodno izmerjenimi eksperimentalnimi rezultati, da smo potrdili točnost razvitega modela. Na koncu je s pomočjo razvitega matematičnega modela bil narejen linearni izračun časovne zgodovine $\mathrm{z}$ uporabo Landersovega spektra pospeškov. Razviti matematični model omogoča preprost in učinkovit izračun potresne odzivnosti lesenih stavb ob upoštevanju razvitih leseno-steklenih stenskih elementov kot nosilnih opornih elementov za horizontalne obremenitve. Model se uporabi tudi v drugih parametričnih numeričnih akademskih študijah, ki analizirajo vpliv različnih parametrov.

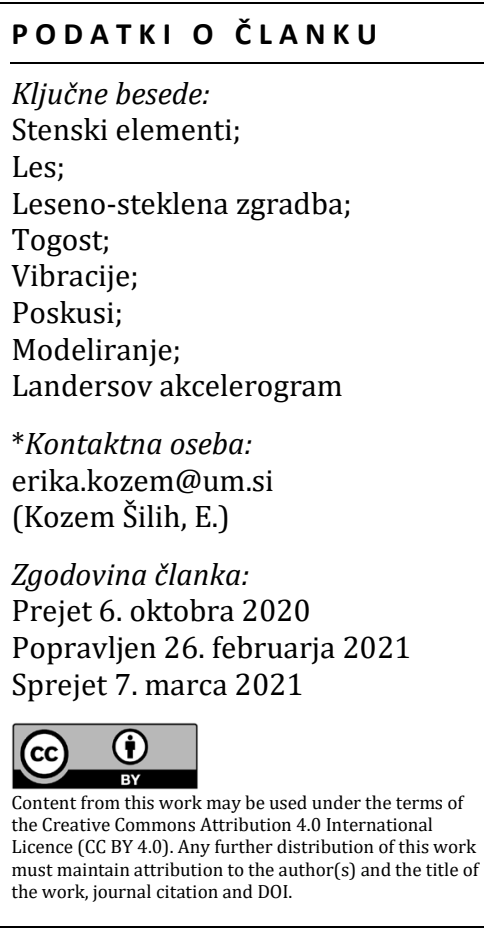

